\title{
Predictors for Tolvaptan Treatment and Future Perspectives
}

\author{
Daisuke NitTA, ${ }^{1}$ MD
}

$\mathrm{H}$ eart failure is a major cause of morbidity and mortality worldwide which poses a great burden not only on the patient but also society owing to its expensive health-care related costs. ${ }^{1)}$ During the past 2 decades, the medication strategies for heart failure have progressed significantly like $\beta$-blockers, angiotensin-converting enzyme inhibitors, angiotensin II receptor blockers, and aldosterone blockers. Although these drugs have somewhat improved the prognosis of heart failure patients, there still remain many patients who suffer from congestive symptoms due to heart failure in spite of high dose diuretics. Tolvaptan (TLV), a unique drug with characteristics of aquaresis via its vasopressin type 2 (V2) receptor blockade, ${ }^{2)}$ is a new drug which was manufactured originally in Japan for heart failure patients. TLV is a promising drug due to its low stimulation of the renin-angiotensin-aldosterone system (RAAS), and prevention of worsening renal function (WRF) and electrolyte balance abnormalities like hyponatremia and hypokalemia. ${ }^{3)}$ Recently, many novel perspectives about the response to and TLV long-term effects of TLV in heart failure have been reported. ${ }^{4-6)}$ Among them, Kadota, et al reported new insights into predictors of a response to TLV by using the arginine-vasopressin (AVP)/plasma aldosterone concentration (PAC) ratio. ${ }^{7)}$ This study was prospectively performed among 26 patients with decompensated heart failure who were treated with $15 \mathrm{mg}$ of TLV. They investigated the effects of TLV on the RAAS and predictors for the response to TLV treatment by using multiple laboratory factors such as plasma renin activity, aldosterone concentration, AVP, and urine osmolality.

TLV increased urine volume and decreased weight without WRF and caused no significant changes in the plasma renin activity or aldosterone concentration. The AVP/PAC ratio before TLV administration was positively correlated with urine volume and they reported the usefulness of the AVP/PAC ratio for predicting the TLV response. Although their study contains some limitations, it provokes important perspectives about prediction of the response to TLV and future challenges in the application of this promising drug.

\section{Article p.461}

Arginine-vasopressin (AVP) is synthesized in the supraoptic and paraventricular nuclei of the hypothalamus and released depending on hyperosmolarity or effective circulation volume loss. Non-osmotic stimulation of AVP is observed, especially among heart failure patients. It was reported that the severity of HF positively correlates with plasma AVP concentration $^{8)}$ and higher AVP is related to a worse outcome. TLV inhibits AVP connection to V2 receptors in the renal collecting ducts and reduces water re-absorption via aquaporin-2 as a consequence. This aquaresis mechanism has many advantages compared to conventional loop diuretics. Loop diuretics are known for their activation of RAAS and decrease the glomerular filtration ratio and activation of the sympathetic nervous system by reducing the effective circulation volume via sodium excretion. ${ }^{3)}$ On the other hand, TLV did not affect serum creatinine and blood urine nitrogen even though its hemoconcentration was the same level and it had no effect on the RAAS because of its depletion of only free water." However, contrary to our expectation, there was no long-term prognostic effect of TLV on mortality among HF patients from the results of the EVEREST trial. ${ }^{10)}$ We realize there are some populations who present no response to TLV therapy, therefore, the next issue is to identify predictors of a response to TLV.

Recently, a few studies have been reported about the predictors of TLV treatment. Among them, Imamura, et al reported that urine osmolality predicted a response to TLV and a higher urine-aquaporin-2/AVP ratio was correlated with the ability of urine concentration of the collecting duct and the usefulness of the urine-aquaporin-2/AVP ratio for the prediction for TLV response. ${ }^{11,12}$ These results mean that the potential ability of urine concentration of the renal collecting duct is essential for the TLV response. High urine osmolality basically indicates high secretion of AVP, but they also reported AVP alone cannot predict a response to TLV. ${ }^{11)}$ The reasons are as follows. First, the low cardiac output and reduction in arterial pressure provoke an inappropriate increase of AVP that is much greater than the level originally needed. Second, as they pointed out, the ability to concentrate urine, which is presented as aquaporin-2, is exasperated among those with chronic kidney disease or long-term low diuretics usage. Thus, the ratio of urine-aquaporin-2/AVP is useful for predicting the actual potency to concentrate urine compared to the secreted AVP alone.

On the other hand, what is the meaning of AVP/PAC as reported by Kadota, et al? The RAAS system is also a significant endocrine system that controls salt-water retention and blood pressure. The primary factors for the stimulation of RAAS are the decreases in blood pressure and sodium concentration. Juxtaglomerular cells in the kidney perceive the decreases in blood pressure and sodium content, and then releas-

From the ${ }^{1}$ Department of Cardiovascular Medicine, Graduate School of Medicine, The University of Tokyo, Tokyo, Japan.

Address for correspondence: Daisuke Nitta, MD, Department of Cardiovascular Medicine, Graduate School of Medicine, The University of Tokyo, 7-3-1 Hongo, Bunkyo-ku, Tokyo 113-8655, Japan. E-mail: dnitsuta-tky@umin.ac.jp

Received for publication May 29, 2016. Accepted June 2, 2016.

Released in advance online on J-STAGE July 11, 2016.

All rights reserved by the International Heart Journal Association. 


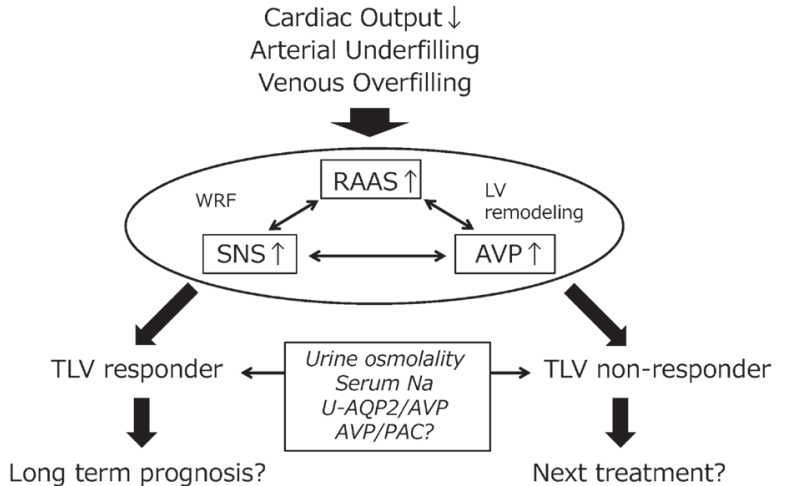

Figure. RAAS indicates renin-angiotensin-aldosterone system; SNS, sympathetic nervous system; AVP, arginine-vasopressin; WRF, worsening renal function; TLV, tolvaptan; U-AQP2, urine aquaporin-2; and PAC, plasma aldosterone concentration.

es renin. Renin converts angiotensinogen to angiotensin I and angiotensin I is converted to angiotensin II by angiotensin converting enzyme. Angiotensin II induces vasoconstriction and stimulates aldosterone secretion in the zona glomerulosa of the adrenal gland. Aldosterone stimulates sodium and water re-absorption in the tubules of the kidney and activates potassium and hydrogen ion secretion. RAAS is also known to be inappropriately elevated among heart failure patients. ${ }^{13)}$ RAAS activation triggers hypokalemia in the tubules of the kidney, which is one of the causes of the secondary renal diabetes insipidus. It was reported that hypokalemia caused a reduction in the intracellular level of cAMP (cyclic adenosine monophosphate), which acts as the secondary messenger of AVP, and downregulated aquaporin-2 in the collecting duct. ${ }^{14)}$ Thus, excessive activation of the RAAS would cause exacerbation of urine concentration via aquaporin-2. Therefore, the ratio of AVP/PAC may reflect the activation of aquaporin-2 in the collecting duct. Larger population studies, including the association of aquaporin-2 and RAAS, should be conducted considering the results of Kadota, et al.

Their study has several limitations. First, their study was too small. There were only 26 patients overall and serum AVP was measured in only 15 patients. Second, they did not compare the incidence of aldosterone blocker between responders and non-responders in Table II. Moreover, they should have compared the AVP/PAC ratio between responders and non-responders. These concerns should be addressed in their next study.

In the current guidelines of heart failure, there are still no statements about TLV administration in responders and nonresponders. The next problem is the long-term prognosis with TLV among the responders, and the further treatment of non- responders. In the future, more detailed guidelines that take into consideration the prediction for responders and the effects for long-term prognosis of TLV should be prepared.

\section{REFERENCES}

1. van Riet EE, Hoes AW, Wagenaar KP, Limburg A, Landman MA, Rutten FH. Epidemiology of heart failure: the prevalence of heart failure and ventricular dysfunction in older adults over time. A systematic review. Eur J Heart Fail 2016; 18: 242-52. (Review)

2. Imamura T, Kinugawa K, Komuro I. Tolvaptan Prolongs Blockage of the Vasopressin Type II Receptor Over 24 Hours in Responders With Stage D Heart Failure. Int Heart J 2016; 57: 41-6.

3. Hirano T, Yamamura Y, Nakamura S, Onogawa T, Mori T. Effects of the V(2)-receptor antagonist OPC-41061 and the loop diuretic furosemide alone and in combination in rats. J Pharmacol Exp Ther 2000; 292: 288-94.

4. Suzuki S, Yoshihisa A, Yamaki T, et al. Vasopressin V2 receptor antagonist tolvaptan is effective in heart failure patients with reduced left ventricular systolic function and low blood pressure. Int Heart J 2015; 56: 213-8.

5. Rosner MH, Ronco C. Hyponatremia in heart failure: the role of arginine vasopressin and its antagonism. Congest Heart Fail 2010; 16: S7-14. (Review)

6. Kinugawa K, Inomata T, Sato N, et al. Effectiveness and adverse events of tolvaptan in octogenarians with heart failure. Interim analyses of Samsca Post-Marketing Surveillance In Heart faiLurE (SMILE study). Int Heart J 2015; 56: 137-43.

7. Kadota M, Ise T, Yagi S, et al. Response Prediction and Influence of Tolvaptan in Chronic Heart Failure Patients Considering the Interaction of the Renin-Angiotensin-Aldosterone System and Arginine Vasopressin. Int Heart J 2016; 57: 461-5.

8. Funayama $\mathrm{H}$, Nakamura $\mathrm{T}$, Saito $\mathrm{T}$, et al. Urinary excretion of aquaporin-2 water channel exaggerated dependent upon vasopressin in congestive heart failure. Kidney Int 2004; 66: 1387-92.

9. Veeraveedu PT, Watanabe K, Ma M, et al. Effects of V2-receptor antagonist tolvaptan and the loop diuretic furosemide in rats with heart failure. Biochem Pharmacol 2008; 75: 1322-30.

10. Konstam MA, Gheorghiade M, Burnett JC Jr, et al. Effects of oral tolvaptan in patients hospitalized for worsening heart failure: the EVEREST Outcome Trial. JAMA 2007; 297: 1319-31.

11. Imamura $\mathrm{T}$, Kinugawa $\mathrm{K}$, Minatsuki $\mathrm{S}$, et al. Urine osmolality estimated using urine urea nitrogen, sodium and creatinine can effectively predict response to tolvaptan in decompensated heart failure patients. Circ J 2013; 77: 1208-13.

12. Imamura $\mathrm{T}$, Kinugawa $\mathrm{K}$, Fujino $\mathrm{T}$, et al. Increased urine aquaporin-2 relative to plasma arginine vasopressin is a novel marker of response to tolvaptan in patients with decompensated heart failure. Circ J 2014; 78: 2240-9.

13. Kumar S, Rubin S, Mather PJ, Whellan DJ. Hyponatremia and vasopressin antagonism in congestive heart failure. Clin Cardiol 2007; 30: 546-51. (Review)

14. Marples D, Frøkiaer J, Dørup J, Knepper MA, Nielsen S. Hypokalemia-induced downregulation of aquaporin-2 water channel expression in rat kidney medulla and cortex. J Clin Invest 1996; 97 : 1960-8. 